\title{
Clinical manifestations and pulmonary
}

\section{histopathological analysis related to different diseases in patients with fatal pulmonary thromboembolism: an autopsy study}

\author{
This article was published in the following Dove Press journal: \\ Open Access Emergency Medicine \\ 10 March 2014 \\ Number of times this article has been viewed
}

\author{
Aline Domingos Pinto \\ Ruppert ${ }^{\prime}$ \\ Alexandre de Matos \\ Soeiro ${ }^{2}$ \\ Maria Carolina Feres de \\ Almeida $^{2}$ \\ Múcio Tavares de Oliveira $\mathrm{Jr}^{2}$ \\ Carlos V Serrano $\mathrm{Jr}^{2}$ \\ Vera Luiza Capelozzi \\ 'Department of Pathology, ${ }^{2}$ Heart \\ Institute, University of São Paulo \\ Medical School, São Paulo, Brazil
}

\begin{abstract}
Background: To correlate underlying diseases, in autopsies of patients with pulmonary thromboembolism (PTE) to histological findings and manifestations reviewed in the medical records.
\end{abstract}

Methods: The autopsy records between 2001 and 2008 of 291 patients whose cause of death was PTE were reviewed. The following data were obtained: age, sex, clinical "in vivo" manifestations, postmortem pathological patterns, and main associated underlying diseases, cancers, and surgeries performed in the last hospitalization. The pulmonary histopathological changes were categorized as diffuse alveolar damage, pulmonary edema, alveolar hemorrhage, and lymphoid interstitial pneumonia. Odds ratios of positive relations were obtained by logistic regression and were considered significant when $P<0.05$.

Results: The median age was 64 years old. About $64 \%$ of patients presented cardiovascular illness associated with PTE. The most prevalent pulmonary finding was pulmonary edema. Only $13 \%$ of cases had clinical suspicion of PTE. Acute respiratory failure was positively related to pulmonary edema, alveolar hemorrhage, and diffuse alveolar damage as well as hemodynamic instability to alveolar hemorrhage and diffuse alveolar damage.

Conclusion: We found important relations between clinical data and histological findings of patients with fatal PTE. A greater understanding of the pulmonary physiopathological mechanisms involved with each disease associated to PTE could improve its diagnosis and treatment.

Keywords: pulmonary embolism, autopsy, pathology, acute respiratory failure, pulmonary edema, fatal

\section{Introduction}

Pulmonary thromboembolism (PTE) is one of the more common immediate causes of death among hospitalized individuals. In general, PTE mortality varies between $6 \%-15 \%$, but when patients present hemodynamic instability or comorbidities, it increases to $20 \%-30 \%$. $^{1,2}$

The epidemiology of PTE has not been fully elucidated, and its nonspecific clinical symptoms make it difficult to establish an accurate diagnosis. Even with the current availability of sophisticated laboratory tests, the real incidence of PTE remains unknown. ${ }^{3}$

Despite the fact that risk factors for PTE are widely reported, pulmonary pathophysiological mechanisms involved in the rapid development of the disease and 
sudden fatal outcomes are unknown. Some autopsy studies have shown that PTE is present in 9\%-21\% of hospital deaths, and in a high number of cases, the finding of PTE is not accompanied by clinical suspicion..$^{4-13}$

In this context, we developed a retrospective study of 291 autopsies with PTE as the postmortem diagnosed cause of death in order to study the prevalence of PTE and to further describe the demographic, clinical, and pulmonary histological data. We seek to expand our understanding of the pathophysiology of sudden onset of fatal PTE, and we have applied basic knowledge regarding lung inflammation and tissue repair, according to the histological results and their correlation with clinical manifestations.

\section{Materials and methods}

\section{Autopsy reports and clinical data}

This study was performed in a tertiary health care center. Between the years 2001 and 2008, 7,661 autopsies were performed and retrospectively reviewed after that. Among them, we included only 291 patients with macroscopic and/or microscopic PTE documented as the main cause of death. All patients with trauma were excluded.

The study was approved by the ethics and research committee, and for all patients in our university center, at least one family member signed an informed consent before the patient was submitted to autopsy. In signing the consent form, the family members acknowledged that the patient could be included in any kind of study at that time or after many years.

We reviewed the medical records and autopsy reports of fatal PTE cases. All medical records were reviewed by the same person according to the protocol. The following data was obtained from the records: age, sex, major diseases, immediate clinical antemortem manifestation, surgery during last hospitalization, and evidence of suspected PTE "in vivo."

Substantial clinical circumstances described in the medical records allowed the categorization of patients according to the following immediate antemortem clinical manifestations: acute respiratory failure (ARF), hemodynamic instability, and sudden death. Sudden death was considered the occurrence of sudden and unexpected death of a patient in apparent stable condition, without premonitory heart failure, myocardial infarction, or other clear cause of death. This included deaths, witnessed or not, in patients who had been seen within 24 hours before death. ${ }^{10-12}$

The syndrome of ARF was defined as having partial pressure of oxygen in the blood $<60 \mathrm{mmHg}$ or partial pressure of carbon dioxide $>50 \mathrm{mmHg}$, with $\mathrm{pH}<7.30$ in ambient air. ${ }^{14-17}$ Arterial blood gas analysis was collected in all patients within 24 hours before death.

Hemodynamic instability was considered when blood pressure fell and required the use of vasoactive drugs or remained low (usually with systolic arterial blood pressure below $90 \mathrm{mmHg}$ or median arterial blood pressure below $70 \mathrm{mmHg}$ after adequate volemic resuscitation) in the last 24 hours before death. ${ }^{14-18}$

The occurrence of clinical suspicion of PTE was considered when there were explicit descriptions in the medical records, even without confirmation of it in vivo.

\section{Pulmonary histological analysis}

Autopsies were performed by resident physicians who followed sequential and systematic, well-established procedures under the supervision of pathologists. Autopsies routinely included topographic analysis and in loco changes, followed by dissection and macroscopic and microscopic analysis of the organs. The protocol was the same throughout 2001-2008.

The diagnosis of PTE was established after confirmation of macroscopic and/or microscopic autopsy, regardless of clinical diagnosis in vivo. In all autopsies, diagnosis of PTE was systematically sought as a protocol. Fatal PTE was considered (and recorded as the cause of death) in the presence of emboli occluding at least two lobar arteries, or even occlusion of interlobar thin branches that generates damage to an extent similar to that of occlusion of at least two lobar arteries, in the absence of other more likely causes of death observed in medical records. ${ }^{19-21}$ In spite of finding that many of the patients had other serious diseases, we only included those patients without a clear in vivo diagnosis described by the physician for their cause of death.

In general, the embolism was considered the main cause of death when it occurred in the main pulmonary trunk or in the proximal right or left pulmonary arteries formed by the bifurcation of the main trunk. ${ }^{18,20}$ Histological analysis of lung was performed in 197 cases of PTE. The histological findings could not be revised in 94 patients because lung tissue was not available for analysis.

In all cases of PTE, the patients were systematically examined for deep vein thrombosis (DVT).

After a complete review, pulmonary pathological findings were classified:

- Diffuse alveolar damage (DAD): diffuse involvement and uniform pulmonary collapse, hyaline membranes, 
obliterative and moderately organized fibrosis and formation of new septa.

- Pulmonary edema (PE): accumulation of proteinaceous fluid in the alveolar spaces.

- Alveolar hemorrhage (AH): presence of blood in the alveolar spaces and intra-alveolar hemosiderin-laden macrophages.

- Lymphoid interstitial pneumonia (LIP): the alveolar are thick and edematous, infiltrated mainly by mononuclear inflammatory cells, lymphocytes, histiocytes, and plasma cells.

All lungs were analyzed by microscopy, even when the reports and findings indicated the diagnosis of the patient. The lungs were fixed in $10 \%$ formalin for at least 4 weeks. We studied a minimum of five slides per lung (total of ten slides per case). Sections of paraffin-embedded tissue were stained with hematoxylin and eosin. To document the presence and distribution of the broad spectrum of infectious agents to which the population is susceptible, we prepared a variety of special stains (periodic acid-Schiff test, immunohistochemistry analysis, fluorescence, Ziehl-Neelsen, Gram, mucicarmine, and Gomori methenamine silver stain) for selected tissue sections.

The occurrence of pulmonary infarction associated with PTE was also observed. Pulmonary infarction was defined as an area of coagulative necrosis of lung tissue, usually as a result of vascular occlusion of the trunk or branches of the pulmonary artery associated with ischemia due to deficiency in systemic arterial circulation. ${ }^{18,20}$

\section{Statistical analysis}

Descriptive analysis of data collected included median, minimum, and maximum values. The odds of PTE comorbidities to develop specific clinical antemortem manifestation described in the medical records or specific histopathological pattern was determined by logistic regression. The variables included major diseases and/or comorbidities: hypertension, dilated cardiomyopathy, chronic obstructive pulmonary disease (COPD), diabetes mellitus, acute myocardial infarction, severe sepsis and/or septic shock, stroke, peripheral arterial obstruction, bronchopneumonia, and cancer.

In addition, we calculated the prevalence of patients undergoing different types of surgery in the last hospitalization (abdominal, neurosurgery, orthopedic, gynecologic, and others) and of different types of neoplasias found in these patients (head and neck, pancreas, intracranial, gynecological, kidney, urinary tract, hematologic, prostate, lung, biliary tract, colon, liver, stomach, and esophagus).
The occurrence of areas of pulmonary infarction associated with PTE was calculated by logistic regression, and this event was correlated with histological patterns, clinical manifestations, and comorbidities.

The relationship was considered statistically significant at $P<0.05$. All statistical procedures were performed using the statistical software SPSS version 10.0 (IBM Corporation, Armonk, NY, USA).

\section{Results \\ Descriptive analysis}

From 2001 to 2008, there was an average of 958 autopsies per year, totaling 7,661 autopsies throughout the 8-year period (42\% of all deaths). PTE was described as a main cause of death in 291 cases (3.8\%) that underwent autopsy and was not clinically suspected in 254 (87\%) of cases. The clinical suspicion of PE was documented in only 37 patients (13\% of cases).

The patients' age ranged from 11-96 years (median 64 years). The demographic findings are shown in Table 1. We observed the description of only one diagnosis coupled with fatal PTE in $55(18.9 \%)$ cases, two diagnoses in $65(22.3 \%)$ cases, three diagnoses in $62(21.3 \%)$, four in $52(178 \%)$, and five in $43(14.7 \%)$ cases. In $14(4.8 \%)$ patients, PTE was the only disease reported in the autopsies. Most of the diagnoses associated with patients with PTE are shown in Table 2. The most prevalent groups of diseases were cardiovascular in $64.26 \%$ of patients, infections in $45.61 \%$, and cancers in $45.02 \%$.

Almost half of the patients who died with PTE had cancer. Among the most prevalent cancers, $13.1 \%$ were digestive, $8.4 \%$ were gynecologic, $5.3 \%$ were urologic, $4.8 \%$ were encephalic, $4.5 \%$ were hematologic, $3.5 \%$ were pulmonary, and $3.1 \%$ were head and neck cancers.

Ninety-one patients $(31.2 \%)$ developed PTE in the postsurgical period of the last hospitalization, mainly related to abdominal surgery $(8.50 \%)$ and obstetric and intracranial surgeries (both 6.8\%).

Table I Demographic findings of patients with pulmonary thromboembolism

\begin{tabular}{llll}
\hline Age (years) & Sex & \multicolumn{1}{l}{ Total } \\
\cline { 2 - 3 } & Female & Male & \\
\hline $0-19$ & $5(71.4 \%)$ & $2(28.6 \%)$ & $7(2.4 \%)$ \\
$20-59$ & $71(60.2 \%)$ & $47(39.8 \%)$ & $118(40.5 \%)$ \\
$60-79$ & $73(55.3 \%)$ & $59(44.7 \%)$ & $132(45.4 \%)$ \\
$\geq 80$ & $15(44.1 \%)$ & $19(55.9 \%)$ & $34(11.7 \%)$ \\
Total & $164(56.4 \%)$ & $127(43.6 \%)$ & 291 \\
\hline
\end{tabular}


Table 2 Underlying diseases and histologic findings in patients with pulmonary thromboembolism

\begin{tabular}{|c|c|c|c|c|c|c|c|}
\hline Disease & DAD & PE & $\mathbf{A H}$ & LIP & Without analysis & Total & (\%) \\
\hline Cancer & $22(16.8 \%)$ & $29(22.1 \%)$ & $28(21.4 \%)$ & $2(1.5 \%)$ & $50(38.2 \%)$ & $|3|$ & 45.02 \\
\hline Systemic arterial hypertension & $9(9.5 \%)$ & $28(29.5 \%)$ & $21(22.1 \%)$ & $4(4.2 \%)$ & $33(34.8 \%)$ & 95 & 32.6 \\
\hline Congestive cardiac insufficiency & $3(3.7 \%)$ & $24(29.3 \%)$ & $26(31.7 \%)$ & $2(2.4 \%)$ & $27(32.9 \%)$ & 82 & 28.18 \\
\hline Bronchopneumonia & $20(27.4 \%)$ & $13(17.8 \%)$ & $17(23.3 \%)$ & $3(4.1 \%)$ & $20(27.4 \%)$ & 73 & 25.09 \\
\hline Sepsis and/or septic shock & $10(23.3 \%)$ & 9 (20.9\%) & 9 (20.9\%) & $2(4.7 \%)$ & $13(30.2 \%)$ & 43 & 14.78 \\
\hline Stroke & $3(13.0 \%)$ & $4(17.4 \%)$ & $4(17.4 \%)$ & I (4.3\%) & II (47.8\%) & 23 & 7.90 \\
\hline Diabetes mellitus & $3(13.0 \%)$ & $4(17.4 \%)$ & $6(26.1 \%)$ & I (4.3\%) & $9(39.1 \%)$ & 23 & 7.90 \\
\hline Acute myocardial infarction & $2(9.1 \%)$ & $5(22.7 \%)$ & $4(18.2 \%)$ & $2(9.1 \%)$ & $9(40.9 \%)$ & 22 & 7.56 \\
\hline COPD & I (5.6\%) & $5(27.8 \%)$ & $4(22.2 \%)$ & $2(11.1 \%)$ & $6(33.3 \%)$ & 18 & 6.19 \\
\hline Acute arterial obstruction member & I (8.3\%) & $3(25 \%)$ & $4(33.3 \%)$ & 0 & $4(33.3 \%)$ & 12 & 4.12 \\
\hline
\end{tabular}

Abbreviations: AH, alveolar hemorrhage; COPD, chronic obstructive pulmonary disease; DAD, diffuse alveolar damage; LIP, lymphoid interstitial pneumonia; $\mathrm{PE}$, pulmonary edema.

The most common clinical manifestation in the period preceding death was ARF, present in 84 cases (28.9\%), followed by sudden death in 80 cases (27.5\%) and hemodynamic instability in $79(26.8 \%)$ cases. The clinical manifestations could not be verified in 48 cases (16.49\%) due to the lack of clear and satisfactory data in the records. We did not perform histological analyses in five of these 48 patients.

The most prevalent histological finding was PE (26.8\%), closely followed by AH (25.4\%), DAD (13.4\%), and LIP (2.4\%). PTE with areas of pulmonary infarction occurred in 47 cases $(16.1 \%)$. The pulmonary analysis showed $22(46.8 \%)$ cases of $\mathrm{AH}$, nine (19.2\%) patients with DAD, and eight patients (17.0\%) with both LIP and PE.

\section{Multivariate analysis}

The correlation between major diseases and histologic patterns is described in Table 3, and the association of diseases and clinical manifestations is shown in Table 4.

Table 5 shows the association of pulmonary infarction and histological patterns. The association between pulmonary infarction and major clinical manifestations showed positive correlation with hemodynamic instability (odds ratio $[\mathrm{OR}]=3.65 ; P=0.05)$ and sudden death $(\mathrm{OR}=5.11$; $P=0.012$ ).

\section{Discussion}

Despite the scientific evidence on the merits of postmortem examination, in modern medical practice its usefulness has been challenged, and the decline in autopsy rates in recent decades has been a worldwide phenomenon. ${ }^{22}$ Studies have shown significant differences between the findings of the autopsy and clinical diagnoses antemortem. ${ }^{22}$ This discordance may vary from $10 \%-90 \%$, depending on the disease and the studied population. ${ }^{22}$ In this study, PTE was not clinically suspected in $87 \%$ of cases, and in accordance with the literature, which describes PTE as one of the major pathological entities not diagnosed in life, was discovered only by postmortem examination. ${ }^{22}$ PTE was responsible for most of the discrepancies, commonly not having been diagnosed correctly in vivo. Moreover, in almost all patients with PTE, the premortem diagnosis was pneumonia. ${ }^{10}$ The lack of clinical suspicion makes

Table 3 Correlation between major diseases and histologic patterns

\begin{tabular}{|c|c|c|c|c|c|c|c|c|c|c|c|c|}
\hline \multirow[t]{2}{*}{ Diseases } & \multicolumn{3}{|c|}{ DAD } & \multicolumn{3}{|l|}{ PE } & \multicolumn{3}{|l|}{$\mathbf{A H}$} & \multicolumn{3}{|l|}{ LIP } \\
\hline & OR & $P$-value & $\mathrm{Cl} 95 \%$ & OR & $P$-value & $\mathrm{Cl} 95 \%$ & OR & $P$-value & $\mathrm{Cl} 95 \%$ & OR & $P$-value & CI $95 \%$ \\
\hline Cancer & 1.52 & NS & $0.56-1.33$ & 1.54 & NS & $0.65-3.54$ & 1.28 & NS & $0.83-2.18$ & 1.77 & NS & $0.38-11.34$ \\
\hline Systemic arterial hypertension & 1.22 & NS & $0.67-2.75$ & 2.3 & NS & $0.83-2.27$ & 1.49 & NS & $0.37-3.89$ & 1.53 & NS & $0.59-4.26$ \\
\hline Congestive cardiac insufficiency & 2.38 & NS & $0.37-6.64$ & 1.37 & NS & $0.42-6.04$ & 1.92 & NS & $0.26-4.44$ & 3.4 & NS & $0.32-4.77$ \\
\hline Bronchopneumonia & 3.15 & NS & $0.53-7.83$ & 1.83 & NS & $0.27-2.39$ & 1.56 & NS & $0.28-6.11$ & 1.06 & NS & $0.75-4.95$ \\
\hline Sepsis and/or septic shock & 1.83 & NS & $0.6-5.65$ & 1.48 & NS & $0.86-4.58$ & 3.27 & NS & $0.38-6.93$ & 2.8 & NS & $0.47-17.93$ \\
\hline Stroke & 1.38 & NS & $0.24-3.89$ & 1.42 & NS & $0.42-2.52$ & 2.34 & NS & $0.08-4.52$ & 2.4 & NS & $0.35-12.48$ \\
\hline Diabetes mellitus & 1.81 & NS & $0.26-5.59$ & 2.53 & NS & $0.64-6.22$ & 1.04 & NS & $0.84-2.55$ & 1.37 & NS & $0.17-3.54$ \\
\hline Acute myocardial infarction & 1.51 & NS & $0.59-4.28$ & 1.84 & NS & $0.26-3.79$ & 1.32 & NS & $0.43-5.30$ & 4.86 & NS & $0.45-29.65$ \\
\hline COPD & 2.73 & NS & $0.45-7.87$ & 2.16 & NS & $0.23-5.35$ & $3.6 I$ & NS & $0.53-4.87$ & 6.42 & 0.02 & $2.84-27.33$ \\
\hline Acute arterial obstruction member & 1.58 & NS & $0.2-3.15$ & 1.94 & NS & $0.25-4.13$ & 1.75 & NS & $0.72-3.71$ & 1.43 & NS & $0.29-11.19$ \\
\hline
\end{tabular}

Abbreviations: $\mathrm{AH}$, alveolar hemorrhage; $\mathrm{Cl}$, confidence interval; COPD, chronic obstructive pulmonary disease; DAD, diffuse alveolar damage; LIP, lymphoid interstitial pneumonia; NS, not statistically significant; OR, odds ratio; PE, pulmonary edema. 
Table 4 Association of diseases and clinical manifestations

\begin{tabular}{|c|c|c|c|c|c|c|c|c|c|}
\hline \multirow[t]{2}{*}{ Diseases } & \multicolumn{3}{|l|}{ ARF } & \multicolumn{3}{|l|}{ HI } & \multicolumn{3}{|l|}{ SD } \\
\hline & OR & $P$-value & Cl $95 \%$ & OR & $P$-value & Cl $95 \%$ & OR & $P$-value & Cl $95 \%$ \\
\hline Cancer & 1.23 & NS & $0.34-3.55$ & 1.44 & NS & $0.53-1.27$ & 2.43 & NS & $0.93-3.81$ \\
\hline Systemic arterial hypertension & 1.36 & NS & $0.4-2.18$ & 1.25 & NS & $0.37-1.99$ & 1.66 & NS & $0.83-3.84$ \\
\hline Congestive cardiac insufficiency & 1.45 & NS & $0.22-5.32$ & 1.73 & NS & $0.86-2.45$ & 2.01 & NS & $0.37-2.98$ \\
\hline Bronchopneumonia & 1.2 & NS & $0.27-3.52$ & 1.5 & NS & $0.29-3.6$ & 1.7 & NS & $0.74-2.98$ \\
\hline Sepsis and/or septic shock & 1.66 & NS & $0.32-3.8$ & 1.32 & NS & $0.93-2.77$ & 1.83 & NS & $0.43-3.42$ \\
\hline Stroke & 1.48 & NS & $0.43-3.22$ & $\mathrm{I} .48$ & NS & $0.65-7.34$ & 2.57 & NS & $0.72-2.98$ \\
\hline Diabetes mellitus & 1.9 & NS & $0.52-3.4 .59$ & 1.61 & NS & $0.5-5.32$ & 1.94 & NS & $0.52-2.85$ \\
\hline Acute myocardial infarction & 1.34 & NS & $0.72-4.72$ & 1.9 & NS & $0.87-2.53$ & 2.36 & NS & $0.44-3.77$ \\
\hline COPD & 1.22 & NS & $0.31-5.62$ & 1.2 & NS & $0.38-5.92$ & 2.4 & NS & $0.89-4.82$ \\
\hline Acute arterial obstruction member & 1.89 & NS & $0.92-4.02$ & 1.78 & NS & $0.80-4.62$ & 4.26 & NS & $0.35-13.7$ \\
\hline
\end{tabular}

Abbreviations: ARF, acute respiratory failure; $\mathrm{Cl}$, confidence interval; COPD, chronic obstructive pulmonary disease; $\mathrm{HI}$, hemodynamic instability; NS, not statistically significant; OR, odds ratio; SD, sudden death.

the early treatment of PTE difficult, which, if established, could reduce mortality.

The fact that PTE in most cases $(\sim 95 \%)$ is associated with one or more diagnoses may contribute to its variable symptoms, complicating the clinical suspicion of PTE. ${ }^{7,8,10,20}$ These discrepancies could be attributed to the nonspecific clinical presentation of PTE and poor quality of care and make evident the importance of postmortem examination.

In our study, PTE was described as a main cause of death in $3.8 \%$ of patients undergoing autopsy. Other autopsy studies (two of them Brazilian studies) showed a prevalence of PTE in approximately $4 \%$ of cases, rates similar to that found in this study. ${ }^{6-8,10,23-25}$

Cardiovascular diseases were related to the PTE in 64.3\% of cases. Among them, stroke and dilated cardiomyopathy are described as important risk factors for thromboembolic events. In the literature, there are studies that relate the ejection fraction and the risk of thromboembolism..$^{26,27}$

DVT was present in $18.2 \%$ of cases. According to the literature, approximately $79 \%$ of PTE patients have evidence of DVT in the lower limbs, and $50 \%$ of patients with DVT have embolization to the lungs. ${ }^{7}$ Our results were lower, probably because DVT was sought only in patients with PTE. In addition, the diagnosis in autopsies have been influenced by many factors of medical interest and are clinically suspicious. Studies have shown that in cases where there was antemortem confirmation of DVT, clinical suspicion of PTE became more frequent. ${ }^{7,23}$

Cancer was the second group of diseases associated with PTE ( $45 \%$ of cases). Patients with cancer suffer more thromboembolism due to several, well-established factors: inflammation (associated with coagulopathy), the use of chemotherapeutic agents, catheter insertion, and possible infectious complications. ${ }^{28-31}$ Cancer is a well-known, major risk factor for PTE, as described in the literature, being more usual in some places than others. ${ }^{28}$ This study reinforces this finding in showing that almost half of the patients who died with PTE also had cancer.

A high prevalence was shown for infectious diseases associated with PTE ( $42.5 \%$ of cases), particularly in patients with respiratory infections (bronchopneumonia was the most prevalent). Tuberculosis, an important disease in Brazil, was present in only ten (3.4\%) patients. ${ }^{8,9,22}$

Only a third of patients $(31.2 \%)$ developed postsurgical PTE in the last hospitalization, which is consistent with some articles in the literature that reveal the occurrence of fatal PTE is more frequent in nonsurgical populations. ${ }^{4}$ However, the clinical suspicion of PTE is most frequent in the postsurgery period. ${ }^{7,23}$

$\mathrm{PE}$ and $\mathrm{AH}$ were the main histological findings in $26.8 \%$ and $25.43 \%$ of cases, respectively. This was probably due to the inclusion of only fatal cases of PTE. This characteristic showed the advanced degree of histologic alterations

Table 5 Multivariate analysis between pulmonary infarction and pulmonary histologic patterns in patients with pulmonary thromboembolism

\begin{tabular}{|c|c|c|c|c|c|c|c|c|c|c|c|c|}
\hline & \multicolumn{3}{|l|}{ PE } & \multicolumn{3}{|l|}{$\mathbf{A H}$} & \multicolumn{3}{|c|}{ DAD } & \multicolumn{3}{|l|}{ LIP } \\
\hline & OR & $P$-value & Cl $95 \%$ & OR & $P$-value & Cl 95\% & OR & $P$-value & Cl $95 \%$ & OR & $P$-value & CI 95\% \\
\hline Pulmonary infarction & 1.92 & NS & $0.86-4.32$ & 3.17 & $<0.001$ & $1.66-6.07$ & 1.69 & NS & $0.74-3.84$ & 4.77 & NS & $0.03-|9.5|$ \\
\hline
\end{tabular}

Abbreviations: $\mathrm{AH}$, alveolar hemorrhage; $\mathrm{Cl}$, confidence interval; DAD, diffuse alveolar damage; LIP, lymphoid interstitial pneumonia; NS, not statistically significant; OR, odds ratio; pulmonary edema. 
observed in the lungs. In addiction, AH was described as one of the major findings, similar to others studies in literature. These histological findings are associated with intracardiac shunt, reduction in the production of pulmonary surfactant, atelectasis and bronchoconstriction after PTE, and severely affect oxygenation. ${ }^{31,32}$

PTE pulmonary infarction occurred in only $16 \%$ of cases and was usually described in patients with preexisting heart or lung disease, which were not correlated in our study. COPD, a disease with systemic inflammatory impact, has been described as an important risk factor for PTE, mainly by blood hyperviscosity. ${ }^{33,34}$

We found a significantly positive relationship between COPD and LIP in cases of fatal PTE (OR 6.42, 95\% CI 2.84-27.33, $P=0.02$ ). However, we observed only two cases of COPD and LIP, and this was a potential bias.

The fact that multivariate analysis did not show a significant relationship between comorbidities simultaneous to PTE and clinical manifestations emphasizes the lack of specific symptoms of PTE and the consequent difficulty in establishing a diagnosis. The symptoms of underlying diseases, viewed independently, are only risk factors, and thus PTE, which may exist quietly and unpredictably, should always be a plausible diagnosis in clinical practice.

The group of infectious diseases had significant statistical relationships with hemodynamic instability and ARF. Infection and associated inflammatory reactions may promote activation of the coagulation cascade, causing PTE. Studies have shown infections, mainly thoracic ones, as risk factors for PTE in hospitalized patients. ${ }^{26}$

Pulmonary infarction, as we expected, significantly increased the risk of AH (OR 3.17, 95\% CI 1.66-6.07, $P<0.001)$. Generally, pulmonary infarcts are hemorrhagic due to the texture of the organ. There was also an association between pulmonary infarction and sudden death (OR 5.11, 95\% CI 1.43-18.25, $P=0.012$ ). As described in the literature, pulmonary infarction occurs mainly in patients with preexisting heart or lung disease, making PTE a more sudden and severe event due to the deficiency in compensatory mechanisms. ${ }^{34,35}$ However, this correlation was not observed in the present study, maybe because the extent of fatal PTE could be responsible for pulmonary infarction, even without comorbidities.

This study had some limitations. First and foremost, this was a retrospective study of medical reports, in which the quality of information may be limited. The second limitation of our study is related to interobserver variability, even taking into account that, at our institution, all autopsies are performed by the resident pathologist, supervised by a senior pathologist who also checks the histological analysis in order to prepare the final reports. Thus, different opinions can be held by different observers. In addition, the accuracy of autopsy findings also depends on the interest and skill of the pathologist, even though a process for systematic analysis of autopsies is in place.

This is the first autopsy study to include clinical and histopathological findings associated with fatal PTE. The most common clinical manifestation was ARF, and cardiovascular diseases were also most prevalent. Underdiagnosed, in vivo PTE remains a cause of a substantial number of deaths. PTE clinical manifestations are unspecific and seem to be connected more to the thrombus and its characteristics (size, extent, location and duration of obstruction, and others). Our results may provide guidance for future studies that seek to discern and evaluate the strengthened related pathophysiological mechanisms of PTE.

\section{Acknowledgment}

This study was supported by FAPESP.

\section{Disclosure}

The authors report no conflicts of interest in this work.

\section{References}

1. Goldhaber SZ, Visani L, De Rosa M. Acute pulmonary embolism: clinical outcomes in the International Cooperative Pulmonary Embolism Registry (ICOPER). Lancet. 1999;353(9162):1386-1389.

2. Carson JL, Kelley MA, Duff A, et al. The clinical course of pulmonary embolism. N Engl J Med. 1992;326(19):1240-1245.

3. PIOPED Investigators. Value of the ventilation/perfusion scan in acute pulmonary embolism: results of the prospective investigation of pulmonary embolism diagnosis (PIOPED). JAMA. 1990;263(20): 2753-2759.

4. Lindblad B, Sternby NH, Bergqvist D. Incidence of venous thromboembolism verified by necropsy over 30 years. BMJ. 1991;302(6778): 709-711.

5. Anderson FA Jr, Wheeler HB, Goldberg RJ, et al. A population-based perspective of the hospital incidence and case-fatality rates of deep vein thrombosis and pulmonary embolism. The Worcester DVT Study. Arch Intern Med. 1991;151(5):933-938.

6. Dismuke SE, Wagner EH. Pulmonary embolism as a cause of death: the changing mortality in hospitalized patients. JAMA. 1986;255(15): 2039-2042.

7. Rubinstein I, Murray D, Hoffstein V. Fatal pulmonary emboli in hospitalized patients: an autopsy study. Arch Intern Med. 1988;148(6): 1425-1426.

8. Menna-Barreto S, Cerski MR, Gazzana MB, Stefani SD, Rossi R. Pulmonary thromboembolism in necropsies at the Clinics Hospital of Porto Alegre. J Bras Pneumol. 1997;23(3):131-136.

9. Alvares F, de Pádua AI, Terra Filho J. [Pulmonary thromboembolism: diagnosis and therapy]. Medicina, Ribeirão Preto. 2003;36(2):214-240. Portuguese.

10. Kotovicz F, Mauad T, Saldiva PH. Clinico-pathological discrepancies in a general university hospital in São Paulo, Brazil. Clinics (Sao Paulo). 2008;63(5):561-568. 
11. Gong DY, Liu XF, Huang FJ. Clinical feature analysis of fatal pulmonary thromboembolism: experiences from 41 autopsy-confirmed cases. Eur Rev Med Pharmacol Sci. 2013;17(5):701-706.

12. Nichols L, Chew B. Causes of sudden unexpected death of adult hospital patients. J Hosp Med. 2012;7(9):706-708.

13. Alfsen GC, Maehlen J. [The value of autopsies for determining the cause of death]. Tidsskr Nor Laegeforen. 2012;132(2):147-151. English, Norwegian.

14. Pouleur AC, Barkoudah E, Uno H, et al; VALIANT Investigators. Pathogenesis of sudden unexpected death in a clinical trial of patients with myocardial infarction and left ventricular dysfunction, heart failure, or both. Circulation. 2010;122(6):597-602.

15. Barbas CS, Capelozzi VL, Hoelz C, et al. [Impact of open lung biopsy on refractory acute respiratory failure]. J Bras Pneumol. 2006;32(5): 418-423. English, Portuguese.

16. Milberg JA, Davis DR, Steinberg KP, Hudson LD. Improved survival of patients with acute respiratory distress syndrome (ARDS): 1983-1993. JAMA. 1995;273(4):306-309.

17. Behrendt CE. Acute respiratory failure in the United States: incidence and 31-day survival. Chest. 2000;118(4):1100-1105.

18. Opinions regarding the diagnosis and management of venous thromboembolic disease. ACCP Consensus Committee on Pulmonary Embolism. American College of Chest Physicians. Chest. 1998;113(2) 499-504

19. Dalen JE, Alpert JS. Natural history of pulmonary embolism. Prog Cardiovasc Dis. 1975;17(4):259-270.

20. Lindblad B, Eriksson A, Bergqvist D. Autopsy-verified pulmonary embolism in a surgical department: analysis of the period from 1951 to 1988. Br J Surg. 1991;78(7):849-852.

21. Yoo HH, De Paiva SA, Silveira LV, Queluz TT. Logistic regression analysis of potential prognostic factors for pulmonary thromboembolism. Chest. 2003;123(3):813-821.

22. Soeiro Ade M, Hovnanian AL, Parra ER, Canzian M, Capelozzi VL. Post-mortem histological pulmonary analysis in patients with HIV/AIDS. Clinics (Sao Paulo). 2008;63(4):497-502.

23. Goldhaber SZ, Hennekens CH, Evans DA, Newton EC, Godleski JJ. Factors associated with correct antemortem diagnosis of pulmonary embolism. Am J Med. 1982;73(6):822-826.
24. Tang Y, Sampson B, Pack S, et al. Ethnic differences in out-of-hospital fatal pulmonary embolism. Circulation. 2011;123(20): 2219-2225.

25. Torbicki A, Perrier A, Konstantinides S, et al; ESC Committee for Practice Guidelines (CPG). Guidelines on the diagnosis and management of acute pulmonary embolism: the Task Force for the Diagnosis and Management of Acute Pulmonary Embolism of the European Society of Cardiology (ESC). Eur Heart J. 2008;29(18):2276-2315.

26. Rocha AT, Paiva EF, Lichtenstein A, Milani R Jr, Cavalheiro CF, Maffei FH. Risk-assessment algorithm and recommendations for venous thromboembolism prophylaxis in medical patients. Vasc Health Risk Manag. 2007;3(4):533-553.

27. Howell MD, Geraci JM, Knowlton AA. Congestive heart failure and outpatient risk of venous thromboembolism: a retrospective, case-control study. J Clin Epidemiol. 2001;54(8):810-816.

28. Woerner EM, Rowe RL. Trousseau's syndrome. Am Fam Physician. 1988;38(3):195-201.

29. Haas S, Wolf H, Kakkar AK, Fareed J, Encke A. Prevention of fatal pulmonary embolism and mortality in surgical patients: a randomized double-blind comparison of LMWH with unfractionated heparin. Thromb Haemost. 2005;94(4):814-819.

30. Kakkar AK, Haas S, Wolf H, Encke A. Evaluation of perioperative fatal pulmonary embolism and death in cancer surgical patients: the MC-4 cancer substudy. Thromb Haemost. 2005;94(4):867-871.

31. Yoo HH, Mendes FG, Alem CE, Fabro AT, Corrente JE, Queluz TT. [Clinicopathological findings in pulmonary thromboembolism: a 24-year autopsy study]. J Bras Pneumol. 2004;30(5):426-432. Portuguese.

32. Rabinovich RA, MacNee W. Chronic obstructive pulmonary disease and its comorbidities. Br J Hosp Med (Lond). 2011;72(3):137-145.

33. Stockley RA. Progression of chronic obstructive pulmonary disease: impact of inflammation, comorbidities and therapeutic intervention. Curr Med Res Opin. 2009;25(5):1235-1245.

34. Alikhan R, Cohen AT, Combe S, et al; MEDENOX Study. Risk factors for venous thromboembolism in hospitalized patients with acute medical illness: analysis of the MEDENOX Study. Arch Intern Med. 2004;164(9):963-968.

35. Pulido T, Aranda A, Zevallos MA, et al. Pulmonary embolism as a cause of death in patients with heart disease: an autopsy study. Chest. 2006;129(5):1282-1287.
Open Access Emergency Medicine

\section{Publish your work in this journal}

Open Access Emergency Medicine is an international, peer-reviewed, open access journal publishing original research, reports, editorials, reviews and commentaries on all aspects of emergency medicine. The manuscript management system is completely online and includes a very quick and fair peer-review system, which is all easy to use.

\section{Dovepress}

Visit http://www.dovepress.com/testimonials.php to read real quotes from published authors. 